\title{
Study on the In vitro and In vivo Antifungal Activities of Nano- silver against Mycoleptodiscus indicus causing Leaf Blight on Lotus in Vietnam
}

Hoang Thi Kim Hong ${ }^{1}$, Nguyen Quang Hoang $\mathrm{Vu}^{2}$, Nguyen Thi Ngoc Hanh ${ }^{3}$, Tran Thi Ha ${ }^{1}$, Le Quang Tien Dung ${ }^{1}$

10.18805/IJARe.AF-685

\begin{abstract}
Background: Lotus, Nelumbo nucifera Gaertn is Vietnam's symbol and Buddhism's flower and plays an essential part in rural Vietnam's economy as all aspects of lotus could bring benefits to farmers. But, unfortunately, lotus yield in Vietnam is seriously affected by various plant diseases. Among them, leaf blight is currently emerging as one of the primary diseases devastating lotus crops in Vietnam, in which there are large necrotic parts on lotus leaves, flowers and seeds.

Methods: Aloe barbadensis extract was used for synthesizing silver nanoparticles. Leaf blight lotus leaves were collected to isolate pathogenic fungi-infection of isolated pathogenic fungi on the healthy lotus and then identify hidden mold by $28 \mathrm{~S}$ rRNA sequencing. Determination of in vitro minimal inhibition concentration of nano-silver was conducted according to Azizi. The reduction of disease symptoms and biological characteristics of the treated lotus was observed.

Result: Morphological analysis and molecular identification of 28S rRNA sequencing showed that the pathogenic microorganism was Mycoleptodiscus indicus (M. indicus). Both in vitro antifungal activity and in vivo treatment of leaf blight lotus using a nano-silver solution showed that $30 \mathrm{ppm}$ of nano-silver was the minimal inhibition concentration (MIC) for totally eradicating $M$. indicus growth. This was the first time $M$. indicus was reported to infect and cause leaf blight on a lotus. Previously, M. indicus was a well-known plant pathogen that could cross-kingdom infect humans and animals. Thus, the fact that lotus is widely cultured in Vietnamese rural could increase the chance for $M$. indicus to spread; hence, this raised the alarm about its potential harm to plants, humans and animals. And, significantly, it revealed nano-silver as a possible approach to prevent $M$. indicus.
\end{abstract}

Key words: Leaf blight, Mycoleptodiscus indicus, Nano-silver, Nelumbo nucifera Gaertn.

\section{INTRODUCTION}

Lotus, Nelumbo nucifera Gaertn, is the symbol of Buddhism. It is also a beautiful and helpful aquatic plant whose whole plant, including flowers, rhizomes, leaves, seeds and young branches. Lotus is used for a wide variety of purposes. Thus, lotus beautifies rural areas and plays an essential part in the rural economy for farmers in places with an abundance of damp soil, ponds and water submerged areas. It is a perennial aquatic macrophyte that has been cultivated for more than 2000 years in Asian countries and Australia, including India, Thailand, Australia, Vietnam, for its economic impacts, cultural values and medicinal uses. Vietnam and India elected lotus as the national flower (Zhu et al., 2019, Mekbib et al., 2020). Lotus was classified as a rhizome lotus with seed lotus and flower lotus based on their specific usages (Guo, 2009). Lotus was reported to perform a high degree of efficiency in photosynthesis and carbon conversion, which leads to a relatively high starch content in its seeds and rhizomes, thus contributing to local cuisine, culture and economy (Zhu et al., 2019).

Leaf blight can affect lotus and other crops, such as maize (Bruns, 2017, DeChant et al., 2017), rice (Chukwu et al., 2019), tomatoes (Adhikari et al., 2017; Esfahani, 2018), lily (Hong et al., 2016), kiwifruit (Adhikari et al. 2017), Litchi chinensis (Kumar et al., 2018), Sansevieria
${ }^{1}$ Hue University of Sciences, Hue University, Vietnam.

${ }^{2}$ Institute of Biotechnology, Hue University Vietnam.

${ }^{3}$ Hong Bang University, Ho Chi Minh, Vietnam.

Corresponding Author: Hoang Thi Kim Hong, Hue University of Sciences, Hue University, Vietnam. Email: hkhong@hueuni.edu.vn

How to cite this article: Kim Hong, H.T., Hoang Vu, N.Q., Ngoc Hanh, N.T., Ha, T.T. and Tien Dung L.Q. (2021). Study on the In vitro and In vivo Antifungal Activities of Nano-silver against Mycoleptodiscus indicus causing Leaf Blight on Lotus in Vietnam. Indian Journal of Agricultural Research. DOI: 10.18805/IJARe.AF-685.

Submitted: 17-08-2021 Accepted: 27-10-2021 Online: 24-11-2021

trifasciata (Kee et al., 2017) and Ixora coccinea (Banerjee et al., 2018). One of the suspected microorganisms causing leaf blight was $M$. indicus, which was reported to infect both plants, animals and humans (Garrison et al., 2008, Dewar and Sigler, 2010, Maboni et al., 2019). On the other hand, overuse of pesticides on leaves was found to leave an alarming amount of harmful residue on the produce, adversely affecting the consumers' health. Thus, there is a need to have a safe solution to prevent leaf blight while not harming the health of humans. To that extent, silver nanoparticles (AgNPs) have increased attention due to their attractive physicochemical properties, especially their anti-microorganism activity. During 
the time while carrying out previous research studies of lotus alkaloids, biodiversity in pink and white lotus in Hue City, Vietnam (Long et al., 2019 Long et al., 2020, Hong et al., 2016, Trang, et al., 2019), researchers observed that leaf bright dramatically affected lotus fields here.

In this study, we synthesized silver nanoparticles using green Aloe barbadensis leaves to study the in vitro and in vivo antifungal activities of nano-silver against Mycoleptodiscus indicus, causing leaf blight on the lotus in Vietnam. We determined the inhibition effects of silver nanoparticles against Mycoleptodiscus indicus species under different concentrations in vitro and in the field trial. This study aims to find an optimal concentration of silver nanoparticles to inhibit the growth and development of Mycoleptodiscus indicus entirely and then use silver nanoparticles to prevent disease on lotus plants.

\section{MATERIALS AND METHODS}

Aloe barbadensis leaves are washed and finely cut into small pieces, then the chopped leaves are put in distilled water and boiled for $15 \mathrm{~min}$, then allowed to cool naturally to room temperature. The solution was filtered and collected for green synthesis of silver nanoparticles and the leaf blight lotus (Nelumbo nucifera) was collected from high yield lotus fields on Hue City, Vietnam.

\section{Green synthesis of silver nanoparticles using Aloe} barbadensis extract

Ten grams of silver nitrate $\left(\mathrm{AgNO}_{3}\right)$ were dissolved in 500 $\mathrm{mL}$ of deionized water. Then, an aqueous solution of silver nitrate was mixed with $500 \mathrm{~mL}$ of plant extract from the Aloe barbadensis. This mixture was stirred for $15 \mathrm{~min}$ at room temperature in a dark condition. Finally, all of the solutions were heated in a microwave $(2,4 \mathrm{GHz}, 800 \mathrm{~W})$. The reduction of $\mathrm{Ag}+$ to $\mathrm{Ag} 0$ was confirmed by the color change of the solution from colorless to yellow, reddish-brown, or UVVisible spectroscopy. Subsequently, the AgNPs solution was cooled down and diluted with $62,5 \mathrm{~L}$ ultrapure water to get an AgNPs solution of 100 ppm. All the AgNPs batches were prepared in duplicate.

\section{Characterization of the silver nanoparticles}

UV-Visible (UV-Vis) absorption measurements were performed using a Shimadzu UV-1800 spectrophotometer (Shimadzu, Kyoto, Japan) from 300 to $700 \mathrm{~nm}$ and operated at a resolution of $1 \mathrm{~nm}$. Scanning electron microscopy (SEM) analysis was used to ascertain the morphology and size of the biosynthesized AgNPs.

\section{Collecting leaf blight lotus leaves}

Leaf blight lotus leaves were collected from a high yield lotus field. Leaves with large necrotic parts were collected. Diseased parts were dry brown, necrotic parts with yellow halos. They had unspecified shapes; some stood as single disease spots while the others might be joined together. Leaf blight lotuses were collected from high yield lotus fields on Hue City, Vietnam.
Young diseased leaves were picked to obtain strong fungal samples.

\section{Isolation of pathogenic fungi}

Small pieces (approximately $2 \times 2 \mathrm{~mm}$ ) of diseased leaf tissue from margins of individual lesions were surface disinfected in $1 \%$ sodium hypochlorite solution for $5 \mathrm{~min}$, rinsed in sterile water, plated on water agar and incubated at $25^{\circ} \mathrm{C}$. After 3 days, mycelium was isolated, transferred to potato dextrose agar (PDA) and then incubated at $25^{\circ} \mathrm{C}$ in a 12-h light/darkness regimen. Pathogenic fungi were isolated and kept in stock on slant PDA (Qiu, Yang et al., 2015, Gurung, Dasila et al., 2020). Fast-growing colonies on PDA were white to orange or pink with abundant acervuli but no perithecium. One-celled conidia were ovoid to oblong and 12 to $20 \times 4$ to $6(15.9 \times 5.0) \mu \mathrm{m}$.

\section{Infection of isolated pathogenic fungi on healthy lotus}

Isolated fungus was cultured in liquid culture. Wounds $1 \mathrm{~cm} \times 1 \mathrm{~cm}$ were made on healthy leaves of a 7-week-old healthy lotus tree. Then these wounds were infected with pathogenic fungal liquid culture. Appearances of leaf blight on lotus were observed (Kim et al., 2001, Choi et al., 2017).

Identification of isolated mold by $28 \mathrm{~S}$ rRNA sequencing

Pathogenic fungi were sent to Nam Khoa Company for molecular identification. After DNA extraction, 28S rRNA sequence was amplified by PCR, aligned and compared to similarities with specific sequences at the National Center for Biotechnology Information (NCBI) (Sandhu et al., 1995).

Determination of in vitro minimal inhibition concentration of nano-silver

An antifungal assay was performed on PDA. After wet sterilization, the PDA was cooled down to $60^{\circ} \mathrm{C}$ and added to an appropriate amount of nano-silver solution to obtain serial dilutions of nano-silver on the PDA (Azizi, Pourseyedi et al., 2016). Then pathogenic fungus was inoculated at 3 points on PDA plates followed by inverted incubation at $30^{\circ} \mathrm{C}-32^{\circ} \mathrm{C}$. Inhibition of nano-silver on the development of pathogenic fungi was observed.

\section{In vivo antifungal activities of nano-silver}

Healthy lotus plants were artificially infected by the pathogenic fungi. When leaf blight appeared, infected lotus were directly sprayed with nano-silver solution at MIC found in previous experiments. The reduction of disease symptoms and biological characteristics of treated lotus was observed (Kim et al., 2001).

\section{Data analysis}

All experiments were triplicated. Data were analyzed by SPSS 20.0.

\section{RESULTS AND DISCUSSION}

\section{Observation of color change and UV-Vis spectroscopy}

The successful synthesis of AgNPs using of Aloe barbadensis extracts were confirmed by color changes and 
Study on the In vitro and In vivo Antifungal Activities of Nano-silver against Mycoleptodiscus indicus causing Leaf Blight on...

spectroscopic analysis. After stirring, the color of the mixture of $\mathrm{AgNO}_{3}$ and plant extracts changed from pale yellow to reddish - brown in $15 \mathrm{~min}$, revealing the conversion of ionic silver $\left(\mathrm{Ag}^{+}\right)$to metallic silver $(\mathrm{Ag})$ and then into colloidal particles (AgNPs). Ag nanoparticles were concentrated and purified by centrifugal ultrafiltration and then rinsed and dried. Formation of $\mathrm{Ag}$ nanoparticles was indicated by theappearance of signature brown colour of the solution (Fig 1). This was the first indication of the efficient synthesis of AgNPs. This observation is consistent with the previous reporting that silver ions are reduced in the presence of plant extracts due to the reducing properties of secondary metabolites (polyphenols, sterols, alkaloids, terpenoids, flavonoids, proteins, etc.).

The mechanism of $\mathrm{Ag}^{+}$reduction reaction to silver nanoparticles is as follow:

$$
\begin{gathered}
4 \mathrm{Ag}^{+}+\mathrm{C}_{6} \mathrm{H}_{5} \mathrm{O}_{7} \mathrm{Na}_{3}+2 \mathrm{H}_{2} \mathrm{O} \rightarrow 4 \mathrm{Ag}^{0}+\mathrm{C}_{6} \mathrm{H}_{5} \mathrm{O}_{7} \mathrm{H}_{3} \\
+3 \mathrm{Na}^{+}+\mathrm{H}^{+}+\mathrm{O}_{2} \uparrow
\end{gathered}
$$

In this reaction, the presence of Aloe barbadensis extract played a role by acting as a stabilizing agent while controlling the silver nanoparticles. Results of electron microscopy analysis showed that silver nanoparticles had quasi-spherical shapes in size range of $5 \mathrm{~nm}-40 \mathrm{~nm}$ (Fig 2).

The wavelength of plant extract nanoparticles was measured using UV-visible Spectroscopy to ascertain the formation of AgNPs. The presence of a strong absorbance peak at about $415 \mathrm{~nm}$ clearly indicated the formation of AgNPs due to the surface plasmon resonance (SPR) electrons phenomena present on the nanoparticle surface (Fig 3 ).

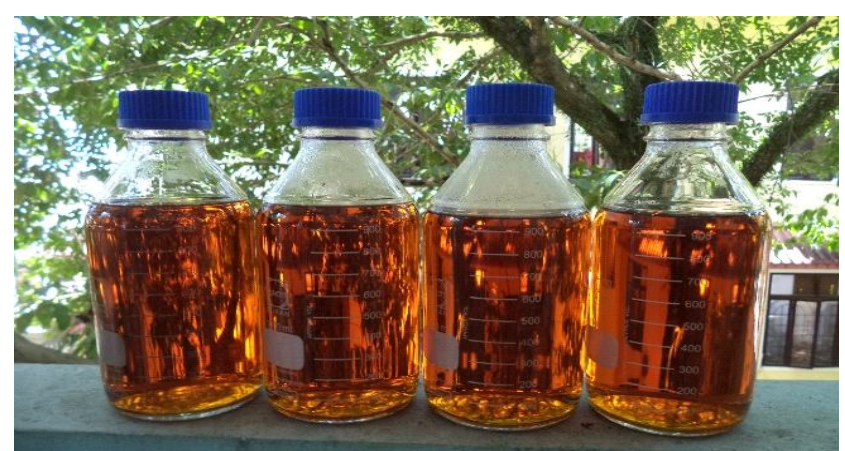

Fig 1: Aqueous synthesized silver nanoparticles.
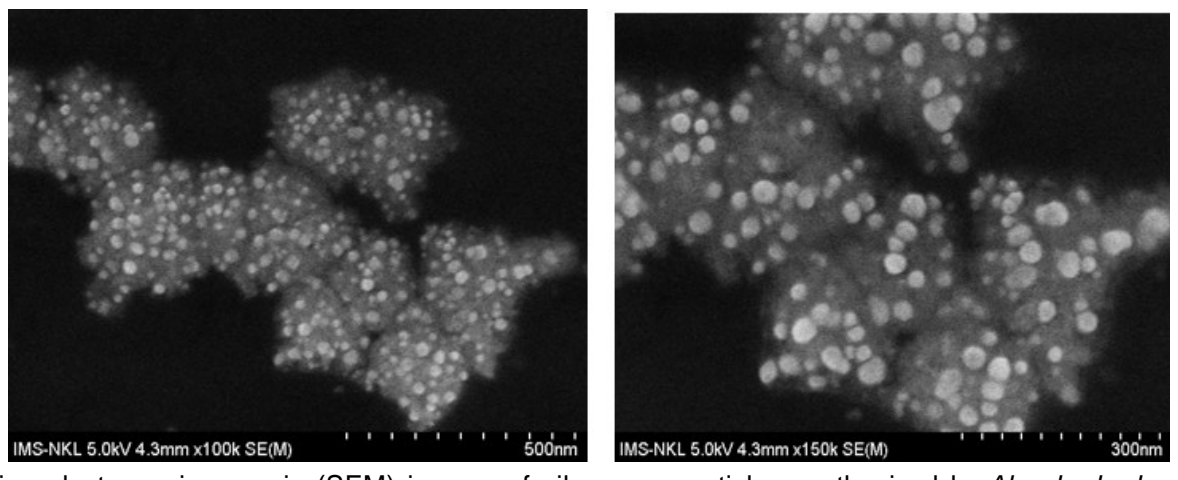

Fig 2: Scanning electron microscopic (SEM) images of silver nanoparticles synthesized by Aloe barbadensis leaf extract.

\section{Morphology of isolated pathogenic fungi}

Leaf blight areas commonly appear at the edge of the leaf veins, or they might spread over the leaf surface (Fig 4). Leaf blight pathogenic microorganisms were isolated on the PDA. Pathogenic fungi formed fast-growing, thick and smooth white mycelia colonies on PDA plates with black spores at the center. Fungal colonies were yellow, as seen from the bottom view (Fig 5A, 5B). Microscopic morphology of isolated fungi showed that this fungus was cellular with branched hyphae. It produced circular spores attached to the end of hyphae (Fig 6A, 6B). The colony morphology and microscopic morphology of the isolated fungi were similar to those of $M$. indicus, which cause rare infections in humans and animals (Maboni, Krimer et al., 2019). Morphology of the fungal colonies was also similar to $M$. indicus isolated from diseased Ixora coccinea (Banerjee, Mandal et al., 2018).

\section{Infection of isolated pathogenic fungi on healthy lotus}

Healthy 7 -week-old lotus leaves were artificially wounded and infected with isolated fungi. After five days, the appearance of leaf blight was observed. The wound spread, turning reddish-brown and the leaf surface became necrotic (Fig 7). The appearance of leaf blight symptoms on the lotus reconfirmed that isolated fungus was the pathogenic cause of this disease on a lotus.

\section{Identification of isolated mold by $28 \mathrm{~S}$ rRNA sequencing}

DNA extraction, PCR for $28 S$ sequencing, analyzed by Basic Local Alignment Search Tool (BLAST) and compared to sequences of those of the NCBI. Those sequences noted were $100 \%$ similar to sequence JE05849, which included 614 nucleotides (score 1134) of $M$. indicus. This finding showed that the isolated pathogenic fungus was $M$. indicus. This was the first time $M$. indicus was reported to cause the leaf blight infection on the lotus (Fig 8).

\section{In vitro antifungal activity and MIC of nano-silver against M. indicus}

People usually use synthetic chemicals to treat diseases on plants and crops. However, this process has led to contamination by harmful residue chemicals of produce. On the other hand, nano-silver is being used to treat deadly

VOLUME ISSUE () 


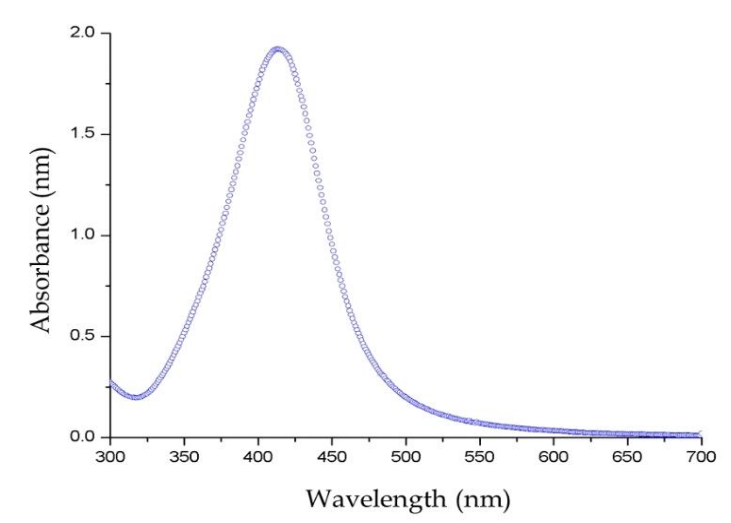

Fig 3: Absorption spectra of nanoparticles. Start wavelength: $300 \mathrm{~nm}$, stop wavelength: $800 \mathrm{~nm}$. diseases on these plants in recent times, which has led to higher yields with less harm to human health. Thus, the choice to treat fungi infections on the edible lotus is a safer solution for leaf blight.

Serial dilutions prepared from nano-silver 40 ppm PDA plates against $\mathrm{M}$. indicus were observed (Fig 9). It was found that increasing silver nano concentration led to more significant and more reliable inhibition of $M$. indicus. From a concentration of $1 \mathrm{ppm}$ to $10 \mathrm{ppm}$, the development of $M$. indicus has slightly reduced this growth compared to the control plate. However, concentrations of nano-silver higher than $15 \mathrm{ppm}$ significantly inhibited $M$. indices' growth. Impressively, $M$. indicus colonies were tiny spots at concentration $25 \mathrm{ppm}$ and they disappeared at the concentration of $30 \mathrm{ppm}$. This finding showed that $30 \mathrm{ppm}$
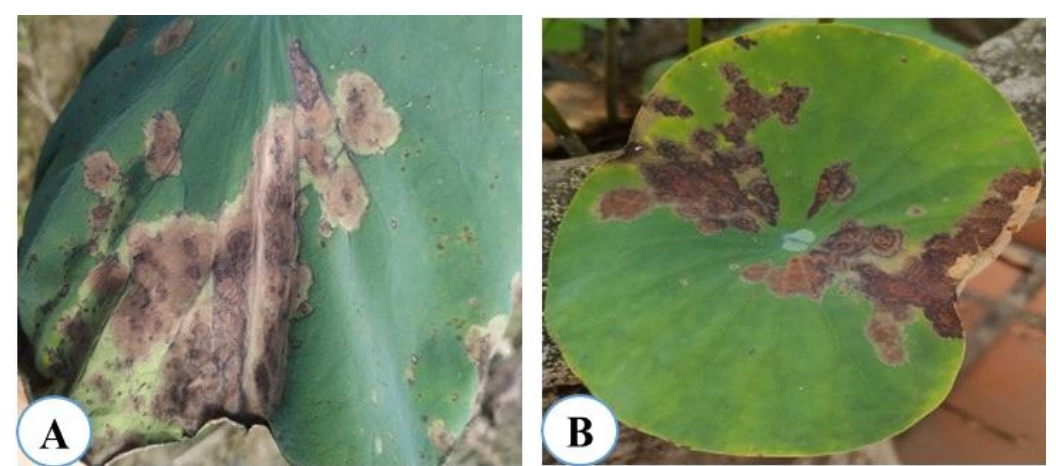

Fig 4: Leaf blight lotus with large necrotic areas in (A) mature leaves and (B) young leaves.
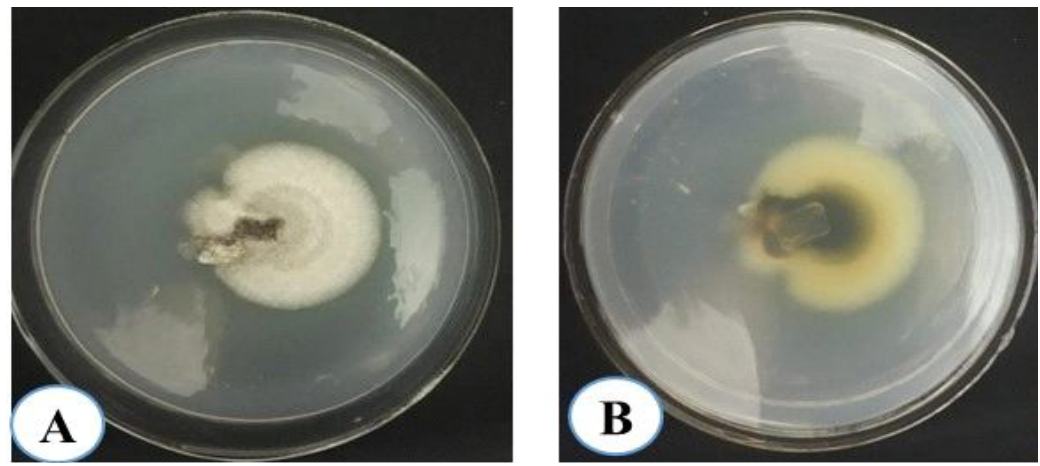

Fig 5: Pathogenic fungal colonies on PDA plates. A. Top view. B. Bottom view.
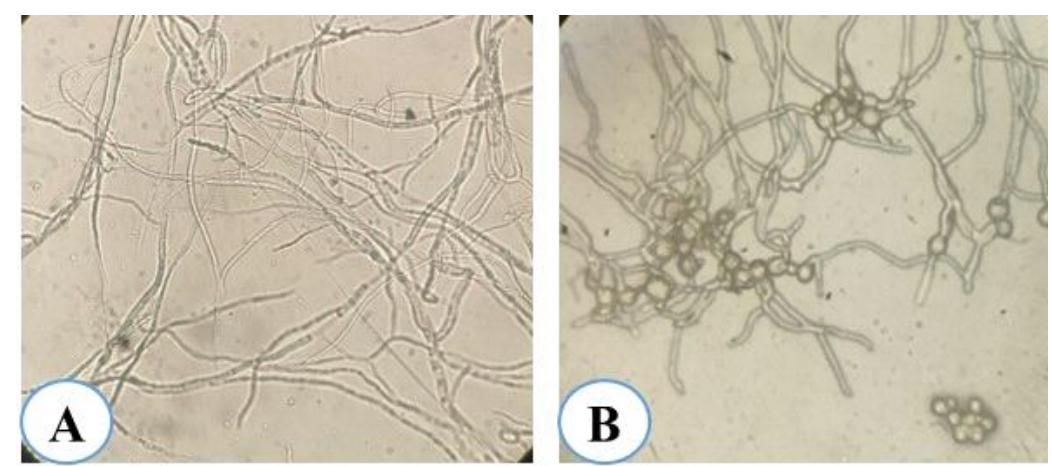

Fig 6: Microscopic morphology of isolated fungi. A. Mycelia (10x40). B. Spore (10x1000). 

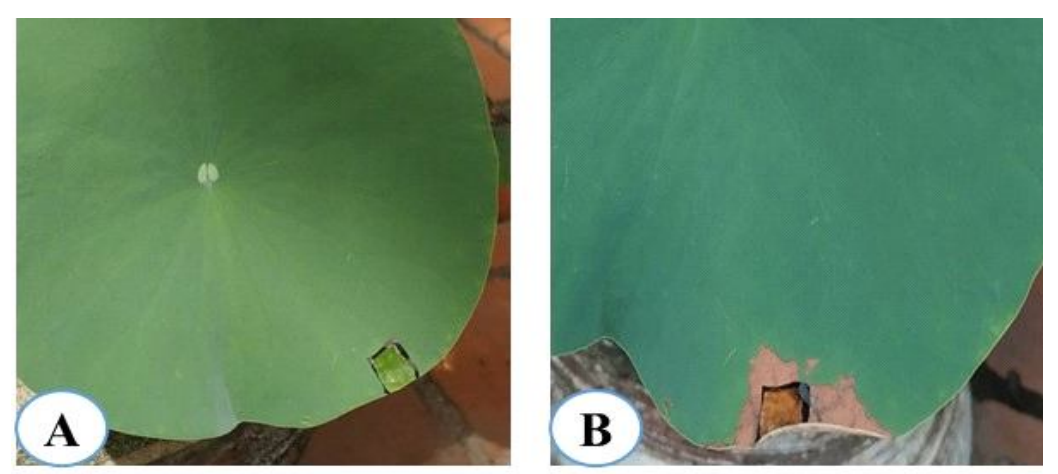

Fig 7: Infection of isolated pathogenic fungi on healthy lotus. A. Wounds on lotus leaves. B. leaf blight appeared after 5 days.

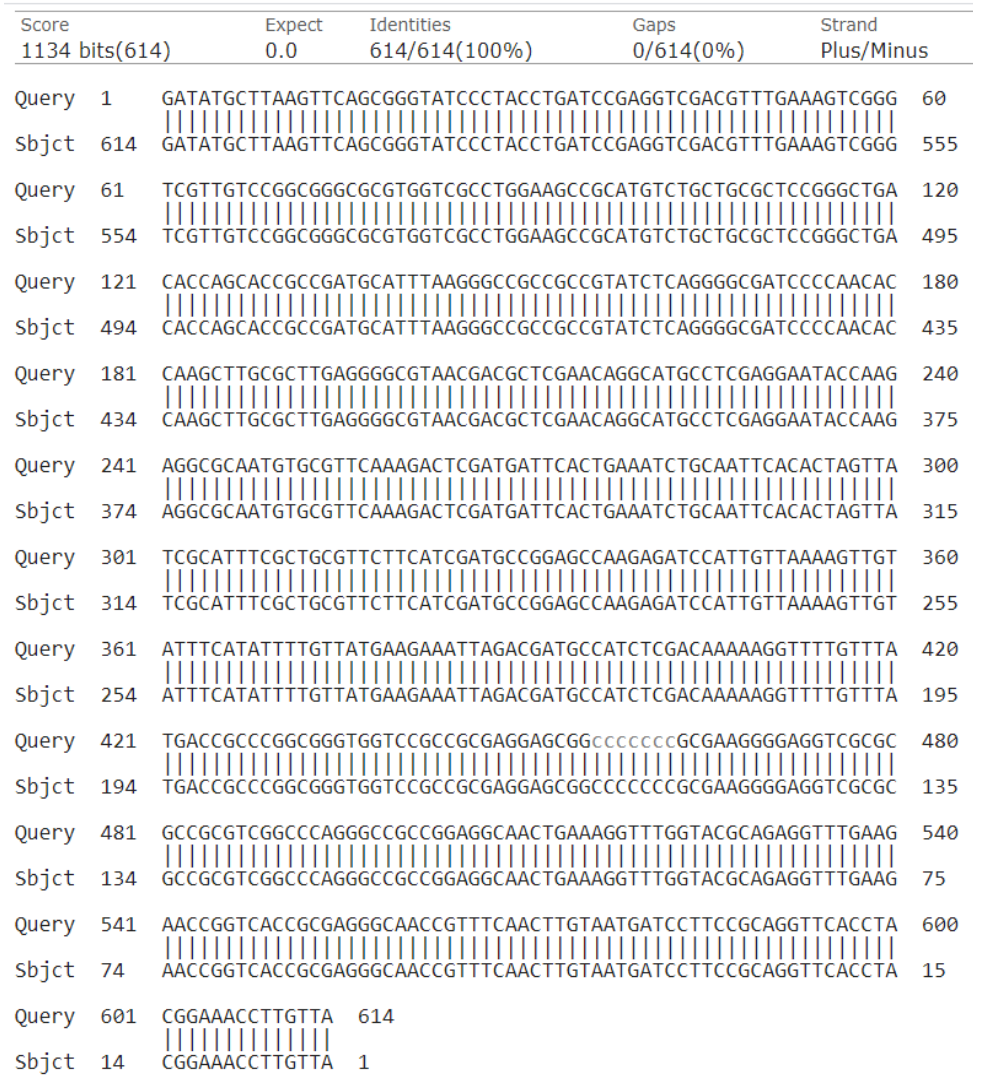

Fig 8: Results from BLAST sequence analysis and sequence on NCBI.

was an in vitro MIC of nano-silver for its inhibition when applied to $M$. indicus.

\section{In vivo antifungal activity of nano-silver}

In vitro MIC of nano-silver - $30 \mathrm{ppm}$ - was applied to treat leaf blight in vivo on artificially induced leaf blight of lotus leaves (Fig 10). Wounds with a size of $1 \mathrm{~cm} \times 1 \mathrm{~cm}$ were made on lotus leaves, followed by spraying of $M$. indicus liquid culture directly on newly wounded areas. The first treatment was 8 hours after infection. Pathogenic symptoms appeared after 8 hours, leading to a spreading of white areas around the initial wound. Next, the 30 ppm of silver nano was sprayed on diseased leaves. Amazingly, the diseased parts did not expand for 24 hours and remained unchanged until five days later. However, after five days, the diseased areas developed necrosis and turned reddishbrown (Fig 10).

On the other hand, the second treatment was carried out 24 hours after developing an $M$. indicus infection. Leaf blight areas (white areas with purple halos) expanded widely on infected leaves after 24 hours of infection. Then this sample was sprayed with $30 \mathrm{ppm}$ nano-silver. The same phenomenon in the previous sample was observed: the leaf blight area stopped expanding and only became necrotic after five days. These impressive inhibitions of leaf blight demonstrated that $30 \mathrm{ppm}$ of nano-silver could 

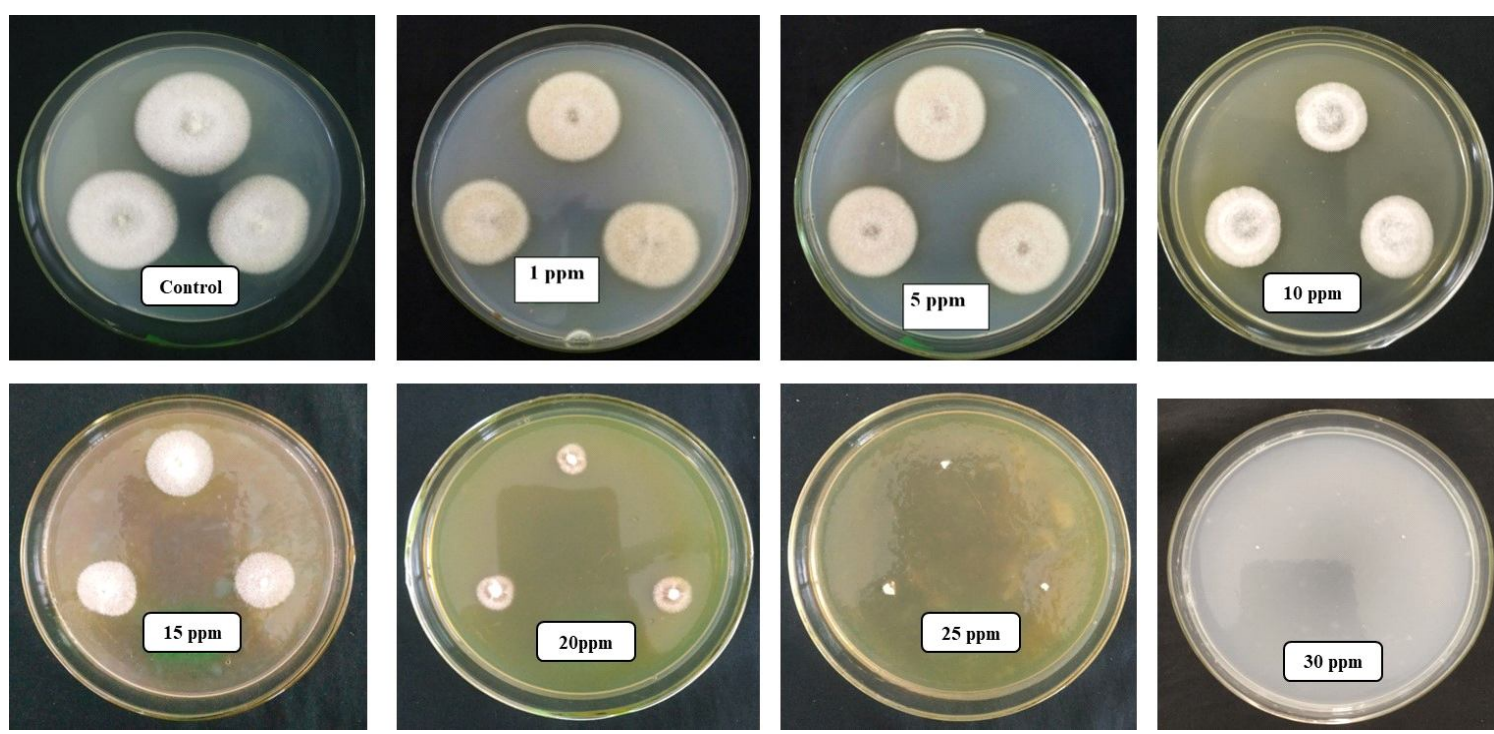

Fig 9: In vitro antifungal activity of nano-silver against $M$. indicus

inhibit the growth of the pathogenic fungi $M$. indicus on lotus leaves.

Mycoleptodiscus indicus is widely known to cause pathogens on many plant parts, causing leaf blight and twig dieback on Ixora coccinea (Ostazeski, 1967, Banerjee et al., 2018). On Zamia, M. indicus caused leaf necrosis with reddish-brown death spots on leaves (El-Gholl and Alfieri, 1991) similar to the necrotic parts on lotus leaves in this current study. Moreover, $M$. indicus is responsible for some diseases on a wide variety of living creatures such as an orchid tree in Malaysia, passion flowers in China, Grape, Ivy, etc.); animals (cats, dogs in the US, etc.) and humans (Maboni et al., 2019). In 2019, an 8 -month-old immunocompetent cat in Georgia, USA, was diagnosed with an infection on the front knee with $M$. indicus (Maboni et al., 2019). It also caused several dermal excoriations in an 8year-old immunodeficient dog (Metry et al., 2010).

Several cases of $M$. indicus infection in humans have been reported. In patients with compromised immune systems, the presence of $M$. indicus can lead to this opportunistic pathogenic microorganism causing an infection. For example, it was observed to cause a proliferation of skin nodules with a sporotrichosis lymphangitic distribution in a 51 -year-old patient who had just undergone liver transplantation and incidentally suffered from a concurrent infection of HIV and HCV. More seriously, this pathogenic fungus invaded his veins and caused an angioinvasion when performed a histologic analysis (Garrison et al., 2008). In 2010, a 54 year old Canadian man was reported to have septic arthritis of his left knee caused by an infection with $M$. indicus after his three-week vacation to Costa Rica (Dewar and Sigler, 2010). In addition, a leg infection with $M$. indicus was found in a 72 - year-old gardener who was also immunodeficient and demonstrated the presence of granulomatosis (Padhye et al., 1995). In 2012, a glioblastoma multiforme patient was also reported
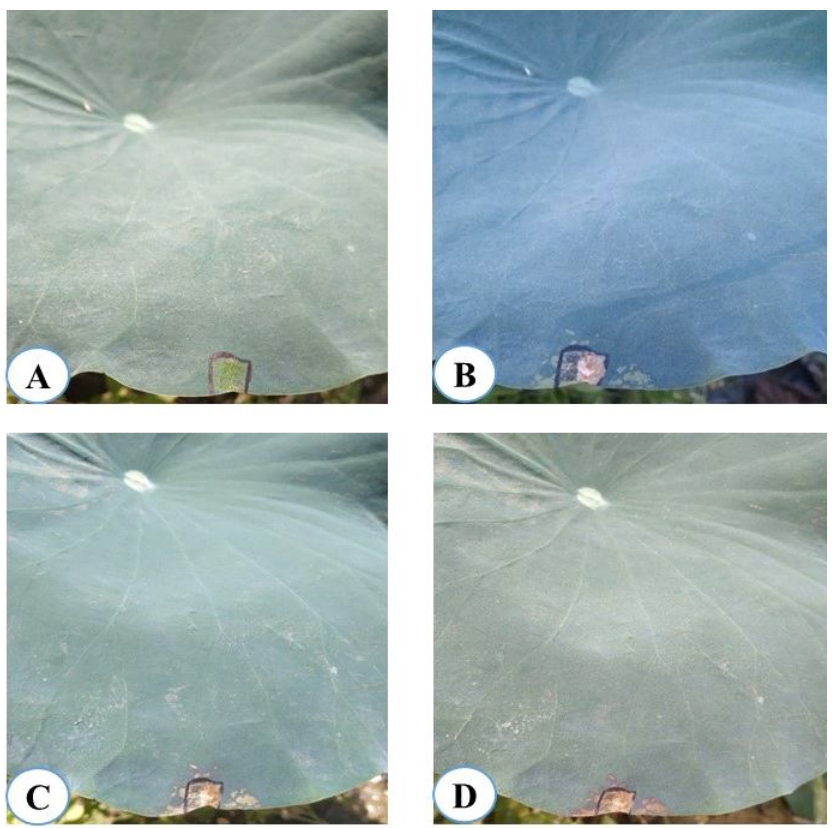

Fig 10: In vivo antifungal activity of nano-silver after 8 hours of $M$. indicus infection. A. wound was made on healthy lotus leaves for the purpose of observing the development of $M$. indicus on them. B. leaf blight appeared after 8 hours. C. leaf blight area after 24 hours treated with $30 \mathrm{ppm}$ nano-silver. D. leaf blight area after 5 days treated with $30 \mathrm{ppm}$ nano-silver.

to be infected with necrotizing lesions of his leg caused by $M$. indicus (Koo et al., 2012). These cases were diagnosed with the awareness of the possibility of $M$. indicus infections in immunodeficient patients who proved to be compatible hosts of this fungus.

Several cases of $M$. indicus infection in humans have been reported. In patients with compromised immune systems, the presence of $M$. indicus can lead to this 
opportunistic pathogenic microorganism causing an infection. For example, it was observed to cause a proliferation of skin nodules with a sporotrichosis lymphangitic distribution in a 51 -year-old patient who had just undergone liver transplantation and incidentally suffered from a concurrent infection of HIV and HCV. More seriously, this pathogenic fungus invaded his veins and caused an angioinvasion when performed a histologic analysis (Garrison et al., 2008). In 2010, a 54-year-old Canadian man reported septic arthritis of his left knee caused by an infection with $M$. indicus after his three-week vacation to Costa Rica (Dewar and Sigler, 2010). In addition, a leg infection with $M$. indicus was found in a 72 - year-old gardener who was also immunodeficient and demonstrated the presence of granulomatosis (Padhye et al., 1995). In 2012, a glioblastoma multiforme patient was also infected with necrotizing lesions of his leg caused by $M$. indicus (Koo et al., 2012). These cases were diagnosed with the awareness of the possibility of $M$. indicus infections in immunodeficient patients who proved to be compatible hosts of this fungus.

\section{CONCLUSION}

$M$. indicus was proven to be the pathogenic microorganism responsible for leaf blight on lotus crops. It is well known as a pathogenic fungus that causes diseases in plants, humans and animals. Fortunately, $30 \mathrm{ppm}$ of nano-silver was found to inhibit its growth in vitro and in vivo. Therefore, it could be concluded that nano-silver might be a potential solution to treating $M$. indicus. However, before a nano-silver solution can be officially applied as a treatment for $M$. indicus, further studies on its activities and safety for humans, plants and animals should be carefully addressed.

\section{ACKNOWLEDGEMENT}

This work was supported by the People's Committee Department of Science and Technology Province ThuaThien Hue, Vietnam for the project (code No: TTH.2019-KC.02). Nguyen Quang Hoang $\mathrm{Vu}$ is sponsored by the Domestic Master/ PhD Scholarship Programme of Vingroup Innovation Foundation (VINIF), Vingroup Big Data Institute (VINBIGDATA), code VINIF.2020.TS89.

\section{REFERENCES}

Adak, T., Swain, H., Munda, S., Mukherjee, A.K., Yadav, M.K., Sundaram, A., Bag, M.K. and Rath, P.C. (2020). Green silver nanoparticles: Synthesis using rice leaf extract, characterization, efficacy and non-target effects. Environmental Science and Pollution Research. 28: 4452-4462.

Adhikari, P., Oh, Y. and Panthee, D.R. (2017). Current status of early blight resistance in tomato: An update. International Journal of Molecular Sciences. 18(10): 1-22.

Azizi, Z., Pourseyedi, S., Khatami, M. and Mohammadi, H. (2016). Stachys lavandulifolia and Lathyrus sp. mediated for green synthesis of silver nanoparticles and evaluation its antifungal activity against Dothiorella sarmentorum. Journal of Cluster Science. 27(5): 1613-1628.
Banerjee, A., Mandal, R. and Nath, P. (2018). First report of leaf blight and twig dieback caused by Mycoleptodiscus indicus on Ixora coccinea. New Disease Reports. 38: 5-8.

Bruns, H.A. (2017). Southern corn leaf blight: A story worth retelling. Agronomy Journal. 109(4): 1218-1224.

Choi N.H., Jang J.Y., Choi G.J., Choi Y.H., Jang K.S., Min B.S., Le Dang Q. and Kim J.C. (2017). Antifungal activity of sterols and dipsacus saponins isolated from Dipsacus asper roots against phytopathogenic fungi. Pesticide Biochemistry and Physiology 141: 103-108.

Chukwu, S., Rafii, M., Ramlee, S., Ismail, S., Hasan, M., Oladosu, Y., Magaji, U., Akos, I. and Olalekan, K. (2019). Bacterial leaf blight resistance in rice: $A$ review of conventional breeding to molecular approach. Molecular Biology Reports. 46(1): 1519-1532.

DeChant, C., Wiesner-Hanks, T., Chen, S., Stewart, E.L., Yosinski, J., Gore, M.A., Nelson, R.J. and Lipson, H. (2017). Automated identification of northern leaf blight-infected maize plants from field imagery using deep learning. Phytopathology. 107(11): 1426-1432.

Dewar, C.L. and Sigler, L. (2010). Fungal arthritis of the knee caused by Mycoleptodiscus indicus. Clinical Rheumatology. 29(9): 1061-1065.

El-Gazzar, N. and Ismail, A.M. (2020). The potential use of Titanium, Silver and Selenium nanoparticles in controlling leaf blight of tomato caused by Alternaria alternata. Biocatalysis and Agricultural Biotechnology. 27: 101-108.

El-Gholl, N. and Alfieri, S. (1991). Leaf necrosis of Zamia caused by Mycoleptodiscus indicus, Fla. Department Agric. and Consumer Serv., Division of Plant Industry. 27.

Esfahani, M.N. (2018). Identification of Ulocladium atrum causing potato leaf blight in Iran. Phytopathologia Mediterranea. 57(1): 112-114.

Garrison, A., Procop, G., Vincek, V., Moon, J., Morris, M., Doblecki, Lewis, S., Cleary, T., Brust, D. and Rosa Cunha, I. (2008). A case of subcutaneous Mycoleptodiscus indicus infection in a liver transplant recipient successfully treated with antifungal therapy. Transplant Infectious Disease. 10(3): 218-220.

Guo, H.B. (2009). Cultivation of lotus (Nelumbo nucifera Gaertn. ssp. nucifera) and its utilization in China. Genetic Resources and Crop Evolution. 56(3): 323-330.

Gurung, K., Dasila, K., Pandey, A. and Bag, N. (2020). Curvularia eragrostidis, a new threat to large cardamom (Amomum subulatum Roxb.) causing leaf blight in Sikkim. Journal of Biosciences. 45(1): 1-8.

Hong, S.J., Koo, T.H. and Yun, S.C. (2016). Controlling Botrytis elliptica leaf blight on hybrid lilies through the application of convergent chemical X-ray irradiation. The Plant Pathology Journal. 32(2): 77.

Kee, Y.J., Suhaimi, N.N., Zakaria, L. and Mohd, M.H. (2017). Characterisation of Neoscytalidium dimidiatum causing leaf blight on Sansevieria trifasciata in Malaysia. Australasian Plant Disease Notes. 12(1): 60.

Kim, J.C., Choi, G.J., Park, J.H., Kim, H.T. and Cho, K.Y. (2001). Activity against plant pathogenic fungi of phomalactone isolated from Nigrospora sphaerica. Pest Management Science: Formerly Pesticide Science. 57(6): 554-559. 
Koo, S., Sutton, D.A., Yeh, W.W., Thompson, E.H., Sigler, L., Shearer, J.F., Hofstra, D.E., Wickes, B.L. and Marty, F.M. (2012). Invasive Mycoleptodiscus fungal cellulitis and myositis. Medical Mycology. 50(7): 740-745.

Kumar, V., Anal, A.K.D., Rai, S. and Nath, V. (2018). Leaf, panicle and fruit blight of litchi (Litchi chinensis) caused by Alternaria alternata in Bihar state, India. Canadian Journal of Plant Pathology. 40(1): 84-89.

Long, D.T., Hong, H.T.K., Tram, L.T.T, Trang, N.T.Q., Tien, N.T.T. and Hanh, N.T.N. (2020). Study of the procedure for bath ultrasound-assisted extraction of total flavonoid from lotus seeds and testing some biological activities. Indian Journal of Agricultural Research. 55(2): 121-128

Long, D.T., Hong, H.T.K., Tram, L.T.T., Trang, N.T.Q., Hiep, N.V. and Tien, N.T.T. (2019). Genetic diversity analysis of lotus species (Nelumbo nucifera gaertn.) in Thua Thien Hue based on ITS4-5 genetic region. Plant Cell Biotechnology and Molecular Biology. 1160-1171.

Maboni, G., Krimer, P., Baptista, R., Lorton, A., Anderson, C. and Sanchez, S. (2019). Laboratory diagnostics, phylogenetic analysis and clinical outcome of a subcutaneous Mycoleptodiscus indicus infection in an immunocompetent cat. BMC Veterinary Research. 15(1): 1-7.

Mekbib, Y., Huang, S.X., Ngarega, B.K., Li, Z.Z., Shi, T., Ou, K.F., Liang, Y.T., Chen, J.M. and Yang, X.Y. (2020). The level of genetic diversity and differentiation of tropical lotus, Nelumbo nucifera Gaertn.(Nelumbonaceae) from Australia, India and Thailand. Botanical Studies. 61: 1-11.

Metry, C.A., Hoien-Dalen, P.S., Maddox, C.W., Thompson, E.H., Sutton, D.A., Romanelli, A.M., Wickes, B.L. and MacNeill, A.L. (2010). Subcutaneous Mycoleptodiscus indicus infection in an immunosuppressed dog. Journal of Clinical Microbiology. 48(8): 3008-3011.
Meza-Menchaca, T., Singh, R.K., Quiroz-Chávez, J., García-Pérez, L.M., Rodríguez-Mora, N., Soto-Luna, M., GastélumContreras, G., Vanzzini-Zago, V., Sharma, L. and QuirozFigueroa, F.R. (2020). First demonstration of clinical fusarium strains causing cross-kingdom infections from humans to plants. Microorganisms. 8(6): 947.

Ostazeski, S.A. (1967). An undescribed fungus associated with a root and crown rot of birdsfoot trefoil (Lotus corniculatus). Mycologia. 59(6): 970-975.

Padhye, A.A., Davis, M.S., Reddick, A., Bell, M.F., Gearhart, E.D. and Von Moll, L. (1995). Mycoleptodiscus indicus: A new etiologic agent of phaeohyphomycosis. Journal of Clinical Microbiology. 33(10): 2796-2797.

Quu, L., Yang, H.H., Wang, L.J., Fan, S.G. and Gao, J.H. (2015). Study on the antibacterial effect of silver nanoparticles on the plant pathogenic fungus Exserohilum turcicum Pass. Applied Mechanics and Materials. 713: 2893-2899.

Sandhu, G.S., Kline, B.C., Stockman, L. and Roberts, G.D. (1995). Molecular probes for diagnosis of fungal infections. Journal of Clinical Microbiology. 33(11): 2913-2919.

Trang, N.T.Q., Thao, T.T.H. and Hong, H.T.K. (2019). Study on the anatomical morphology of lotus varieties (Nelumbo nucifera gaertn.) in Vietnam. Plant Cell Biotechnology and Molecular Biology. 95-105.

Zhu, F., Sun, H., Diao, Y., Zheng, X., Xie, K. and Hu, Z. (2019). Genetic diversity, functional properties and expression analysis of NnSBE genes involved in starch synthesis of lotus (Nelumbo nucifera Gaertn.). Peer J. 7: e7750. 